\title{
On the microscopic structure of liquid hydrogens
}

\author{
M.Zoppi ${ }^{1}$, M.Celli ${ }^{1}$, U.Bafile ${ }^{1}$, E.Guarini ${ }^{2}$, M.Neumann ${ }^{3}$ \\ 1 Consiglio Nazionale delle Ricerche, Istituto di Elettronica Quantistica, \\ Via Panciatichi 56/30, I-50127 Firenze, Italy \\ 2 Istituto Nazionale di Fisica della Materia, Unitá di Genova, \\ Dipartimento di Fisica, Genova, Italy \\ 3 Institut für Experimentalphysik der Universität Wien, \\ Strudlhofgasse 4, A-1090 Wien, Austria
}

Received August 23, 2000

\begin{abstract}
Among the quantum liquids, the hydrogens cover an interesting position between liquid helium, where quantum exchange gives rise to the macroscopic phenomenon of superfluidity, and neon, where quantum effects are relatively small, so that its features can be evaluated by perturbation methods with reference to a classical system. Nonetheless, the experimental access to the microscopic structure of the hydrogens is not an easy task both because of their intra-molecular structure and the small molecular mass that is comparable with that of the neutron probe. In this paper we discuss the state of the art and summarise the available experimental information on the microscopic structure of the hydrogens. The experimental data for the two systems are compared among them and with the results of quantum Path Integral Monte Carlo simulations. It is found that similar quantities, measured in corresponding thermodynamic points, are rather different for the two systems due to the different weight of quantum effects. Moreover, the comparison with the simulation results shows that, for deuterium, there is a substantial agreement, both at the level of the structure factor and its thermodynamic derivatives. The agreement is less satisfactory for liquid hydrogen.
\end{abstract}

Key words: quantum liquids, liquid hydrogen, quantum effects

PACS: $61.25 . E m, 61.12 . G z$

\section{Introduction}

Quantum effects, in condensed matter, originate from the de-localization of the particles which cannot be associated with individual points in the space but are characterised by a probability distribution [1]. This, in turn, is somehow bound to the de Broglie thermal wavelength, $\lambda_{\mathrm{DB}}$, which depends on the mass of the molecule 
Table 1. Evaluation of quantum effects for common quantum liquids. The subscript CP and TP refer to the Critical Point and to the Triple Point, respectively. For helium, TP indicates the $\lambda$-point. $\lambda_{\mathrm{DB}}$ is the de Broglie wavelength defined in equation 1 . The parameters $\sigma$ and $\ell$ represent the hard core diameter of the particle and the average interparticle distance, respectively.

\begin{tabular}{|c|rr|rc|cc|cc|}
\hline System & \multicolumn{1}{|c|}{$\begin{array}{c}T_{\mathrm{CP}} \\
(\mathrm{K})\end{array}$} & $\begin{array}{c}T_{\mathrm{TP}} \\
(\mathrm{K})\end{array}$ & $\begin{array}{c}n_{\mathrm{CP}} \\
\left(\mathrm{nm}^{-3}\right)\end{array}$ & $\begin{array}{c}n_{\mathrm{TP}} \\
\left(\mathrm{nm}^{-3}\right)\end{array}$ & $\left(\frac{\lambda_{\mathrm{DB}}}{\sigma}\right)_{\mathrm{CP}}$ & $\left(\frac{\lambda_{\mathrm{DB}}}{\sigma}\right)_{\mathrm{TP}}$ & $\left(\frac{\lambda_{\mathrm{DB}}}{\ell}\right)_{\mathrm{CP}}\left(\frac{\lambda_{\mathrm{DB}}}{\ell}\right)_{\mathrm{TP}}$ \\
\hline $\mathrm{He}$ & 5.20 & 2.18 & 10.47 & 21.99 & 1.50 & 2.31 & 0.84 & 1.66 \\
$\mathrm{H}_{2}$ & 33.19 & 13.96 & 9.00 & 23.06 & 0.72 & 1.11 & 0.44 & 0.94 \\
$\mathrm{D}_{2}$ & 38.34 & 18.71 & 10.44 & 25.99 & 0.47 & 0.68 & 0.31 & 0.60 \\
$\mathrm{Ne}$ & 44.4 & 24.55 & 14.31 & 37.2 & 0.21 & 0.29 & 0.14 & 0.26 \\
\hline
\end{tabular}

and on the temperature, according to the definition [2] :

$$
\lambda_{\mathrm{DB}}=h /\left(2 \pi M k_{\mathrm{B}} T\right)^{1 / 2},
$$

where $M$ is the mass, $T$ is the temperature, and $k_{\mathrm{B}}$ is the Boltzmann constant. Strictly speaking, the wavelength associated with a free massive particle can only be defined assuming that the momentum of the quantum particle, $p=M v$, is identified with the momentum of the associated plane wave, $p=\hbar k=h / \lambda$. This would imply a slightly different definition for the thermal wavelength.

For a dense system of interacting particles, the relation connecting $\lambda_{\mathrm{DB}}$ (or any similarly defined thermal wavelength) with the space probability distribution is not trivial. In addition, the actual size of the space probability distribution gives rise to different effects, depending on the value of its ratio with the two other length scales, namely the molecular size and the interparticle distance. If we define $\sigma_{\mathrm{CM}}$ as the full width of the space probability distribution of the molecular centres of mass, this quantity should be compared with $\sigma$, the usual hard-core parameter of the pair potential, and with $\ell$, the average interparticle distance. As a 0-th order approximation, we can assume that $\sigma_{\mathrm{CM}} \approx \lambda_{\mathrm{DB}}$. Thus, the relevant quantities can be calculated and are reported in table 1 .

Depending on the different ratios between $\lambda_{\mathrm{DB}}$ and $\sigma$, or between $\lambda_{\mathrm{DB}}$ and $\ell$, substantially different quantum effects are observed. For liquid helium, $\lambda_{\mathrm{DB}}$ is larger than $\ell$. This allows a quantum exchange between neighbouring particles and produces the macroscopic effect of superfluidity. However, as the density decreases (e.g. around the critical point), the ratio becomes $<1$ and the quantum exchange is quenched. Nonetheless, the quantum size of the particle is sensibly larger than $\sigma$ and therefore large quantum effects are still expected due to the de-localization of the particles (quantum diffraction). On going from helium to hydrogen, and deuterium, and neon, the expected exchange effects are even smaller. We see that for deuterium and neon, the value of $\lambda_{\mathrm{DB}}$ never exceeds the size of $\sigma$ and, to reproduce the experimental data, it is sufficient to take into account the quantum diffraction effect only. For neon, this ratio is so small that the expected quantum effects can 
be effectively taken into account using perturbation theories on a classical reference system [3]. For the hydrogens, instead, the quantum diffraction features are expected to be rather large $\left(\lambda_{\mathrm{DB}}\right.$ exceeds the value of $\sigma$ ) but no quantum exchange effect is expected for those systems. In fact, even for liquid hydrogen at the lowest temperature, i.e. close to the triple point, the ratio between $\lambda_{\mathrm{DB}}$ and $\ell$ is $<1$. This ratio could increase by decreasing the temperature, but in this case the liquid freezes and, due to the zero-point motion of the molecular centres of mass, the kinetic energy increases and so does the effective temperature.

To sum up, we observe that the hydrogens, among the quantum liquids, play a very special role. On the one hand, the exchange effects appear to be negligible, and therefore the Boltzmann statistics can be applied. On the other hand, the quantum diffraction effects, i.e. those due to the spatial broadening of the centre-of-mass wavefunction, are quite relevant and cannot be evaluated by a simple perturbation theory. In addition, due to the same electronic structure, the hydrogens are expected to experience, at least to a first approximation, the same intermolecular potential. Therefore, the different behaviour that is observed in the two systems can only be attributed to a different size of the quantum effects that, in turn, are determined by the different molecular mass.

From the experimental point of view, to determine the microscopic structure of liquid hydrogens is not a trivial task. On the one hand, the small number of electrons make the hydrogens almost transparent to X-rays. The alternative experimental technique, namely the neutron diffraction, turns out to be difficult too. The small molecular mass, comparable with that of the probe, makes the inelasticity effects quite relevant and this implies that the neutron diffraction data are heavily affected by inelasticity effects. In addition, among the hydrogens, there is a substantial difference between the two isotopes. For deuterium, in fact, the coherent neutron cross section, i.e. the parameter that is in front of the microscopic structure factor in the scattering cross section, is of the same order of magnitude of the incoherent one. This implies that, qualitatively, the interesting signal lies on top of a background that is of a similar order of magnitude [4]. For hydrogen, instead, the ratio between the incoherent and the coherent cross sections is almost two orders of magnitude. This makes the required signal extremely small, with respect to the incoherent background, and explains why no generally accepted experimental structure factor exists, to date, for liquid hydrogen.

We are confident that a great part of the experimental problems could be dealt with in a not too far future. Many experimental groups are working on it (see, for example, [5]) and small partial achievements are obtained, month after month. However, the interpretation of the experimental results needs also a reliable theoretical framework that is necessary to extract the relevant information from the experimental spectra. Dealing with quantum liquids is certainly more complex than with the classical ones. As a matter of fact, theories to evaluate the microscopic structure of simple classical liquids have reached a satisfactory level [2]. For quantum liquids, instead, the theoretical approach is still in its infancy, even though the Path Integral Monte Carlo (PIMC) simulation technique can be used to compute, given a suitable 
intermolecular potential, the microscopic structure of monatomic quantum liquids $[6,7]$. The technique was initially implemented to simulate Boltzmann particles, and has been recently improved to take into account the quantum statistics as well. For example, using a PIMC simulation technique that is able to take into account the Bose-Einstein statistics, Ceperley has shown that beautiful results can be obtained for the microscopic structure of superfluid liquid helium [8]. Of course, dealing with a Boltzmann quantum liquid is much less demanding, from a computational point of view.

The microscopic structure of the quantum liquids listed in table 1 is affected by quantum diffraction effects depending both on the temperature and the molecular mass. For monatomic liquids, like helium or neon, the calculation of the microscopic structure is more direct than for the molecular liquids. However, as the hydrogens are characterised by a simple intramolecular structure, it is sufficient to take into account the many-body quantum effects on the centre-of-mass distribution and to deal with the intramolecular structure using suitable models. As a consequence, in order to compare the experimental results with the theory, one has to combine the results of the PIMC simulations (centres of mass structure) with those obtained from a suitable theoretical calculation of the intramolecular structure.

We observe that, for a homonuclear diatomic molecule, the diffraction cross section can be expressed as [4]:

$$
\frac{\mathrm{d} \sigma}{\mathrm{d} \Omega}=u(Q)[S(Q)-1]+v(Q)+P(Q),
$$

where $S(Q)$ is the static structure factor of the molecular centres of mass and the functions $u(Q)$ and $v(Q)$ are molecular form factors which are interpreted as the intermolecular and intramolecular neutron cross sections respectively. The unknown function $P(Q)$ accounts for the inelastic scattering corrections. We point out that in writing equation 2 we totally neglect the orientational correlations. This is a reasonable assumption for liquid hydrogens, as it is well known that this is well verified even in the solid phase [9].

If we apply a free rigid-rotor model to the molecule, then the expressions for the molecular form factors assume a very simple analytical form [10]. However, it is well known that this simple model is insufficient to take into account, quantitatively, the microscopic structure of deuterium and should be generalised [4]. Young and Koppel [11] have shown that, in the gas phase, a free rotating harmonic oscillator model gives a substantially correct description of the neutron cross section of the hydrogens. Therefore, to a first approximation, the molecule can be modelled as a freely rotating harmonic oscillator. In this case, the molecular form factors can be calculated and are expressed as a sequence of functions approximating the true behaviour [12].

To the lowest order, the functions $u(Q)$ and $v(Q)$ become the familiar rigid rotor functions that are now modulated by a Debye-Waller factor generated by the harmonic oscillator [12]. These are:

$$
u^{(0)}(Q)=4 a_{\mathrm{coh}}^{2}\left[\exp \left(-\frac{\lambda_{\mathrm{DW}}^{2} Q^{2}}{2}\right) \frac{\sin \left(Q D_{\mathrm{e}} / 2\right)}{\left(Q D_{\mathrm{e}} / 2\right)}\right]^{2}
$$


and

$$
v^{(0)}(Q)=2\left(a_{\mathrm{coh}}^{2}+a_{\mathrm{inc}}^{2}\right)+2\left(a_{\mathrm{coh}}^{2}-b a_{\mathrm{inc}}^{2}\right) \exp \left(-2 \lambda_{\mathrm{DW}}^{2} Q^{2}\right) \frac{\sin \left(Q D_{\mathrm{e}}\right)}{\left(Q D_{\mathrm{e}}\right)},
$$

where $\lambda_{\mathrm{DW}}=\left(\hbar / 2 M \omega_{\mathrm{v}}\right)^{1 / 2}$ is the Debye-Waller wavelength and $\omega_{\mathrm{v}}$ is the circular frequency of the molecular vibration. The parameter $D_{\mathrm{e}}$ represents the average equilibrium distance of the two nuclei while $a_{\text {coh }}$ and $a_{\text {inc }}$ represent the coherent and incoherent scattering length [13]. For orthodeuterium $b=0$, while for parahydrogen $b=1[12]$. The sequences converge rapidly and it is found that their limiting behaviour can be well represented by equations 3 and 4 if the parameters $\lambda_{\mathrm{DW}}$ and $D_{\mathrm{e}}$ are replaced by slightly different effective parameters [12].

\section{An overview of the experimental results on the $S(Q)$}

The experimental determination of the structure factor of helium, over a wide range of thermodynamic state points, is rather old [14] and that of liquid neon is even older [15]. Instead, no reliable information was available on liquid hydrogens till recently [16]. In fact, in order to correct the neutron diffraction data for inelasticity effects, researchers have mostly used the so-called Placzek correction procedure [17]. This is a perturbative treatment whose critical parameter is the ratio between the mass of the neutron and that of the target particle. For helium, this value is $1 / 4$ and the theory is still manageable. However, the theory holds for a monatomic system and could only be applied to a molecular system by introducing the concept of effective mass (atomic mass $\leqslant m_{\mathrm{E}} \leqslant$ molecular mass) which in turn depends on the energy of the incident neutrons. For hydrogen, the mass ratio would become, at best, $1 / 2$ which makes the Placzek correction procedure unmanageable.

The turning point was determined by the availability of new diffraction instruments that use a pulsed neutron source instead of a reactor. In fact, on a reactor source, the incident neutrons are of constant energy and the momentum transfer, $\hbar Q$, is changed simply by changing the scattering angle. On the contrary, using a pulsed source, the scattering angle is kept fixed and the spread in $Q$ is obtained from the energy distribution of the incident neutrons. Thus, there is an intrinsic advantage in using time-of-flight (TOF) neutron diffraction for measuring the static structure factor $S(Q)$ of liquids composed of light molecules. This is simply related to the fact that, here, a complete information can be obtained maintaining the scattering angle to a rather small value. As the inelasticity effects increase by increasing the scattering angle, it appears that choosing a sufficiently small scattering angle the inelasticity effects can be kept within an acceptable level, even for a light system like hydrogen.

The first measure of the microscopic structure of deuterium gas on a standard powder diffractometer convinced us that the problem of the inelastic scattering corrections could be greatly reduced using only the small angle scattering detectors [18]. Following this achievement, we took advantage of the construction of a dedicated liquid diffractometer (SANDALS, at ISIS, UK) that uses an almost forward scattering 


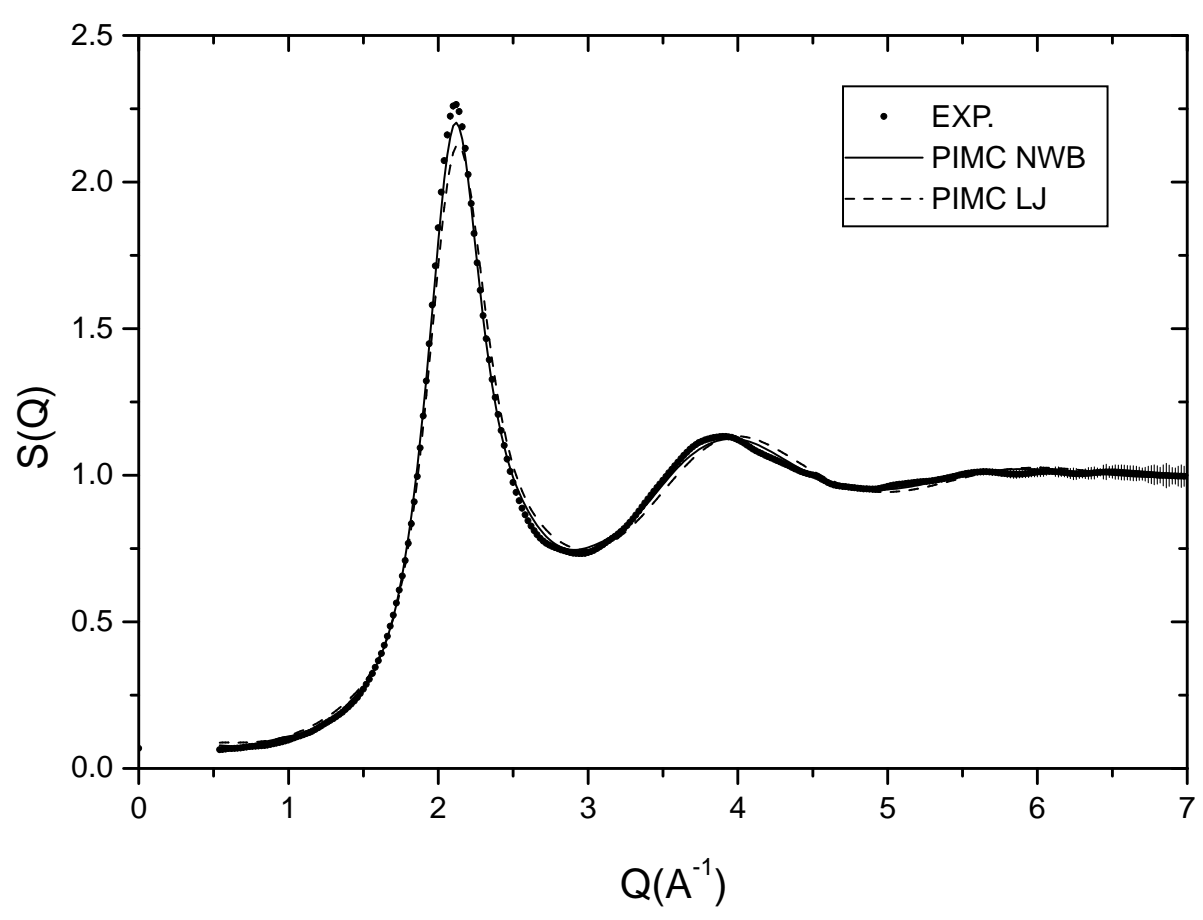

Figure 1. Microscopic structure factor for the centres of mass distribution of liquid deuterium close to the Triple Point. The dots with the error bars (almost invisible in the figure) are the experimental points, while the lines represent the PIMC simulation results using two different intermolecular potentials. The continuous line is obtained using the NWB phenomenological potential, while the dashed line represents the Lennard Jones results.

geometry. Using SANDALS, the structure factor of liquid deuterium was measured both in the vicinity of the triple point [4] and close to the freezing transition [19].

Once the know-how was established, a diffraction experiment on liquid deuterium was also carried out at a reactor source [20], which allowed us to refine the experimental results. In fact, for the two experiments, the inelasticity corrections are located in different spectral regions and therefore, from a critical comparison of the two experimental determinations, we were able to obtain an accurate determination of the structure factor of liquid deuterium which, in turn could be positively compared with the simulation results [21]. This comparison is reported in figure 1. Here, the experiment is compared with the PIMC simulation results obtained using either the Norman, Watts, and Buck (NWB) [22] or the Lennard Jones (LJ) [23] intermolecular potential (cf. the continuous and the dashed lines in figure 1).

Thus the residual difficulty for performing an experiment on hydrogen is the unfavourable ratio between the coherent and the incoherent cross section. Because of this ratio, the coherent scattering contribution, which carries the information on the intermolecular structure, is expected to become almost invisible due to the overwhelming incoherent background. In fact, a rough calculation [12] assuming that the intermolecular (centre of mass) structure factor of liquid $\mathrm{H}_{2}$ is similar to that of $D_{2}$, would predict a coherent modulation of the order of $4 \%$ of the incoherent 
background. So, the experiment appears to be intrinsically difficult, also because we expect that the coherent response of hydrogen will be smaller than that of deuterium due to the larger quantum effects induced by the smaller molecular mass. However, the situation improves if pure para-hydrogen is considered. In this case, due to the decrease of the intramolecular scattering term at low $Q$ for para-hydrogen, this ratio would becomes $10 \%$ in the region of the main peak of the structure factor and the experiment would appear feasible, with a reasonable accuracy.

Based on this premise we have carried out a neutron diffraction experiment on liquid para-hydrogen on SANDALS. However, as we will show in the following section, the predictions were too optimistic and it was impossible to extract a reliable microscopic structure factor from the large incoherent background.

One might argue that, in the end, an experimental determination of the microscopic structure factor of hydrogen is not strictly necessary. A sufficiently accurate $S(Q)$ has been determined for deuterium and, using a suitable intermolecular potential, it was shown that PIMC simulations could reproduce the experimental structure factor. Therefore, the hydrogen structure factor could be determined, to a good approximation, using the same potential and a PIMC simulation applied to hydrogen.

On the other hand, there is a number of questions that are waiting for an answer. For example, to which extent the hydrogen and deuterium intermolecular potentials can be considered equal? Is it not possible that the quantum mechanical $g(r)$ (or $S(Q)$ ) can be obtained from the classical ones simply by a convolution with a suitable single-particle (center of mass) distribution function? Of course, the present state of the art of the PIMC technique can give an answer to these and other questions, but the final assessment, in our opinion, can only rely on the comparison with solid experimental data. For this reason only the experimental results can definitely answer these and similar questions.

\section{Thermodynamic derivatives of $S(Q)$}

Even though the microscopic structure factor represents the final aim of our experimental efforts, the lack of information on the $S(Q)$ of hydrogen does not mean that additional experimental information could not be obtained from the experiments.

In fact, considering equation 2 , we observe that the most relevant dependence on the thermodynamic conditions of the sample is in $S(Q)$. Therefore, since our experimental data were taken in selected thermodynamic points (cf. table 2), and by assuming that $P(Q)$ is constant, we could derive an experimental determination of the thermodynamic derivatives of $S(Q)$. This procedure was successfully used for liquid deuterium data close to the triple point $[4,20]$.

However, the same procedure could not be applied to the hydrogen data. In fact, the size of the inelastic scattering corrections, that is expected to be proportional to the total cross section, becomes now relevant. In other words, due to the large intramolecular contribution to the scattering cross section, even small fluctuations 
Table 2. Details of the thermodynamic conditions of the experiments. The estimated error on the temperature is $0.1 \mathrm{~K}$, for the $\mathrm{H} 2$ experiment, and $0.5 \mathrm{~K}$, for the D2 experiment. The uncertainty on the pressure is 0.1 bar. As a consequence, the error on the density of hydrogen is $0.03 \mathrm{~nm}^{-3}$ and that on the density of deuterium turns out twice as large.

\begin{tabular}{|c|ccc|}
\hline State & $T(\mathrm{~K})$ & $p($ bar $)$ & $n\left(\mathrm{~nm}^{-3}\right)$ \\
\hline $\mathrm{D} 2-1$ & 20.7 & 2.2 & 25.42 \\
$\mathrm{D} 2-2$ & 20.7 & 20.1 & 25.84 \\
$\mathrm{D} 2-3$ & 22.0 & 20.2 & 25.45 \\
$\mathrm{D} 2-4$ & 20.7 & 40.2 & 26.30 \\
$\mathrm{D} 2-5$ & 23.5 & 40.6 & 25.46 \\
& & & \\
$\mathrm{H} 2-1$ & 17.1 & 2.0 & 22.22 \\
$\mathrm{H} 2-2$ & 17.1 & 29.9 & 23.00 \\
$\mathrm{H} 2-3$ & 20.3 & 29.9 & 22.22 \\
$\mathrm{H} 2-4$ & 17.1 & 16.2 & 22.61 \\
$\mathrm{H} 2-5$ & 18.6 & 15.9 & 22.19 \\
\hline
\end{tabular}

in the instrument electronics become now crucial and tend to generate noise that submerges the coherent signal. For these reasons, a different procedure was used for hydrogen [24]. Making use of the experimental differences, and of equation 2 to remove the molecular form factor, we were finally able to obtain the thermodynamic derivatives of the structure factor $S(Q)$.

In figure 2 we report $[\partial S(Q) / \partial n]_{T}$, the experimental density derivative, at constant temperature, of the hydrogens. As it was anticipated, the deuterium determination appears more precise than that of hydrogen. The two sets of data are qualitatively and quantitatively very similar, even though small differences can be observed from a superposition of the two figures. The experimental temperature derivative at constant density, $[\partial S(Q) / \partial T]_{n}$, is reported in figure 3 . Again, the deuterium case is more definite and the two figures are qualitatively very similar. However, some quantitative difference can be observed, especially in the position of the main minimum.

A quantitative comparison between the two sets of data is reported in the two following figures. In figure 4 the deuterium data for the density derivative are represented by the continuous line (a spline fit through the experimental points) while the hydrogen data are the points with the error bars. The quantitative comparison is rather good, even though the hydrogen data seem to suggest a slight shift to smaller $Q$-values. In addition, some difference appears in the small- $Q$ region where the hydrogen data seem to rise faster to the zero-level. In figure 5, we report the temperature derivative (the symbols have the same meaning) but now the shift of the hydrogen data toward smaller $Q$-values appears more evident than before.

While for the first observation, namely the shift that is observed in both figures 


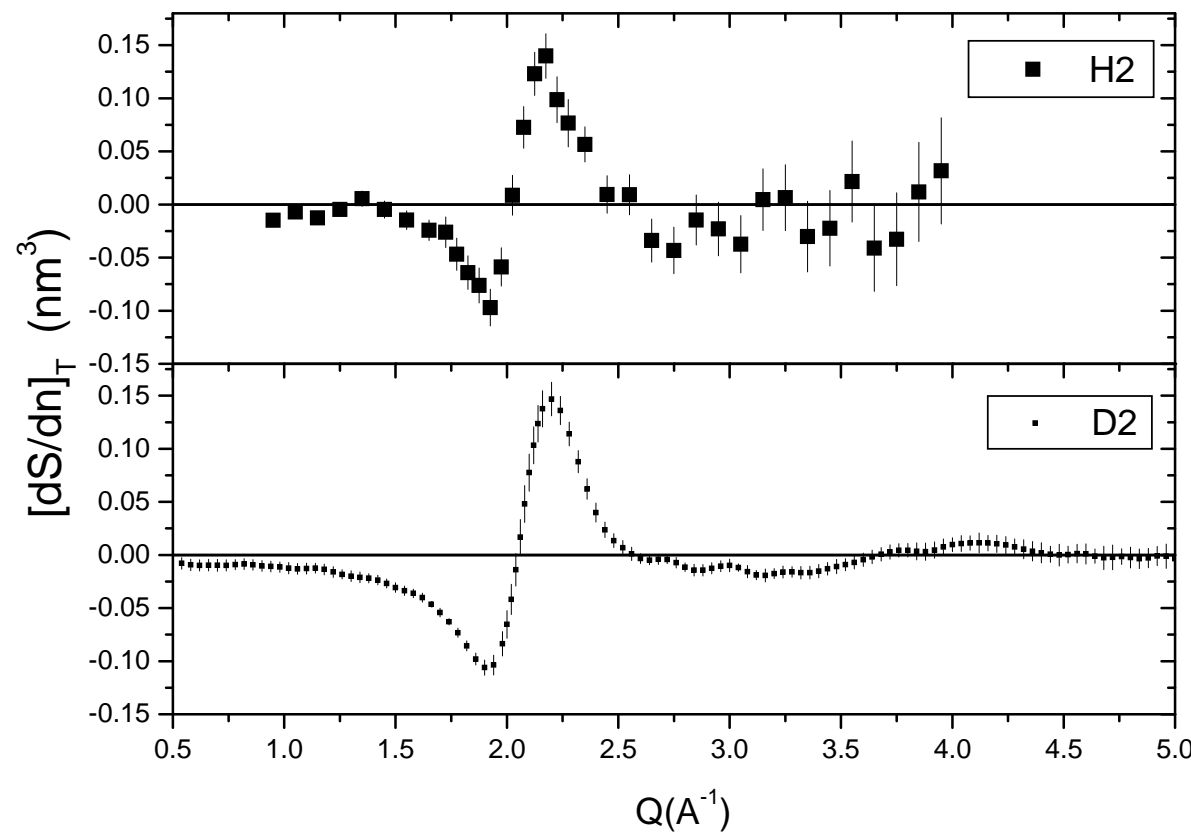

Figure 2. Density derivative, at constant temperature, of the structure factor of the hydrogens. The two pictures appear quantitatively similar, even though the deuterium data (lower figure) appear much cleaner that the hydrogen ones (upper figure).

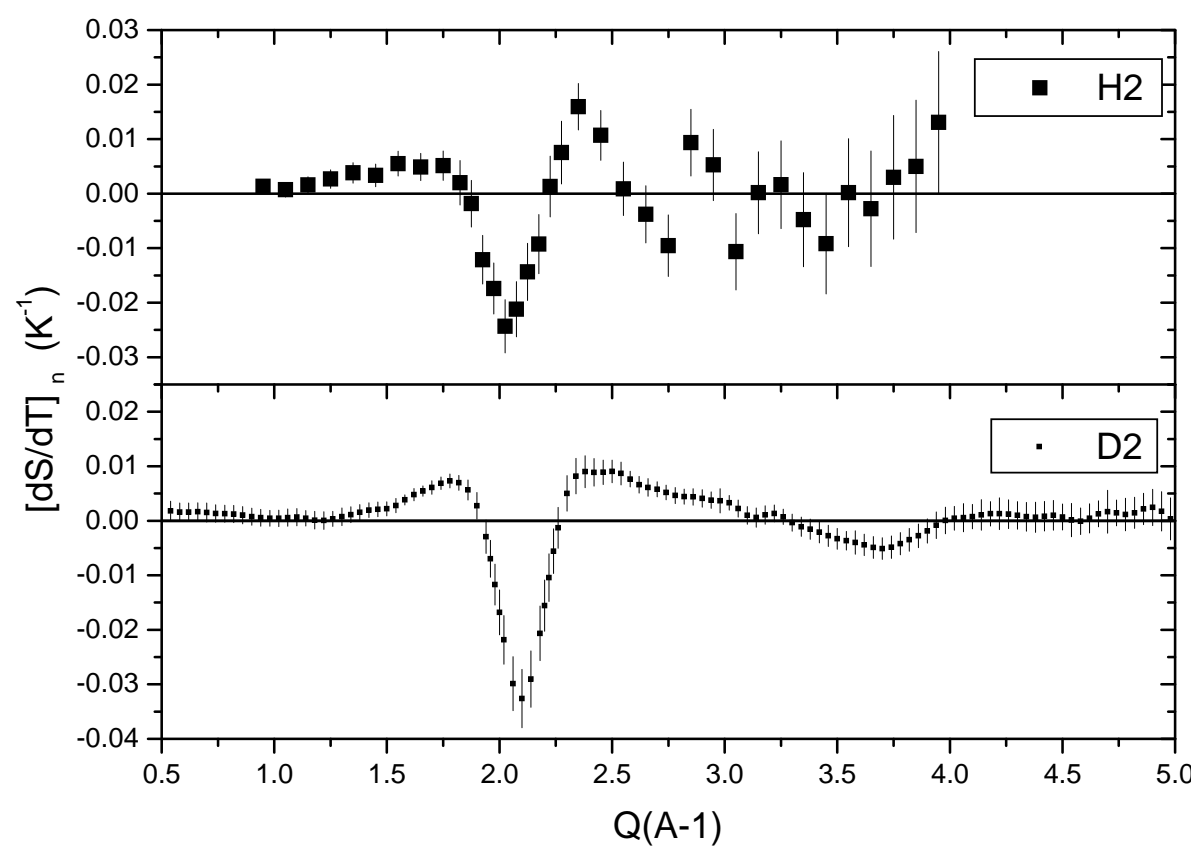

Figure 3. Temperature derivative, at constant density, of the structure factor of the hydrogens. Also in this case the two pictures appear quantitatively similar. However, a slight difference can be observed in the depth of the minimum that appears larger for deuterium. 


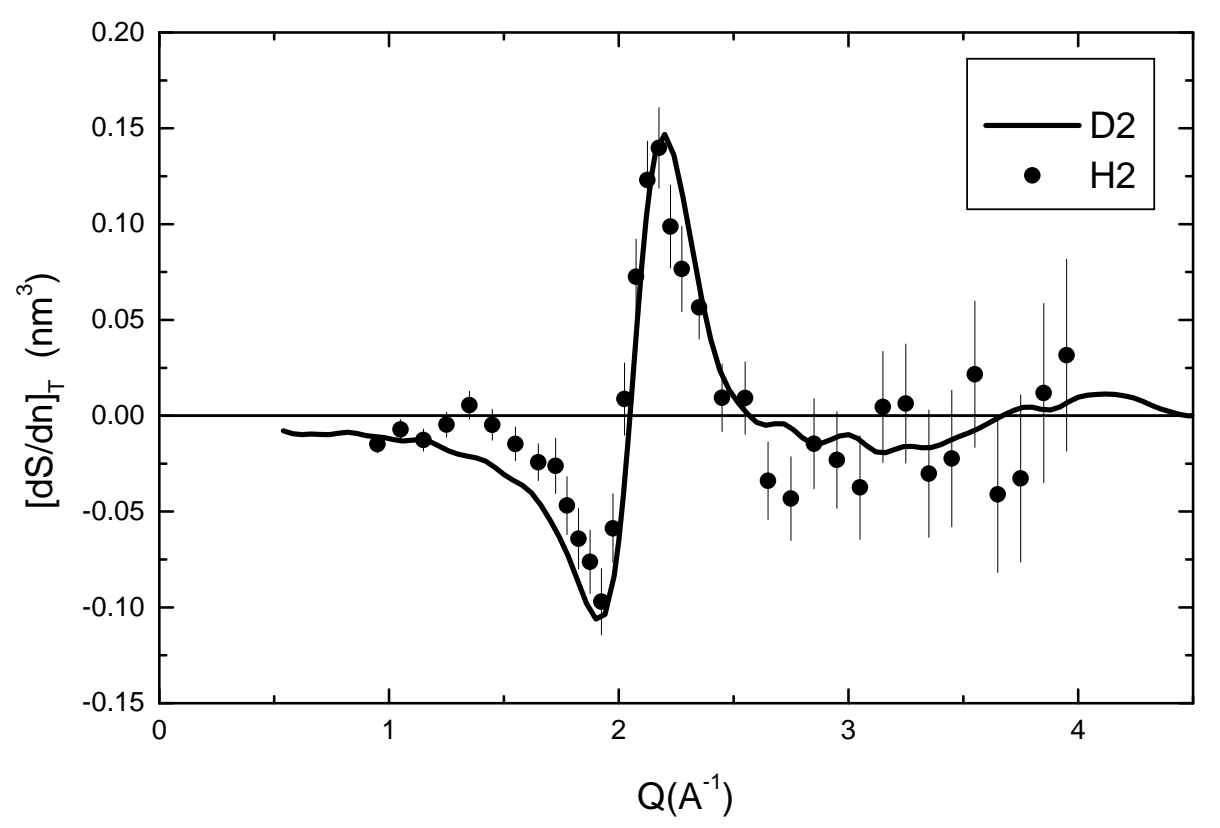

Figure 4. Direct comparison between the density derivatives of $S(Q)$ of the hydrogens. The line represents the deuterium behaviour while the hydrogen data are represented by the black dots with the error bars.

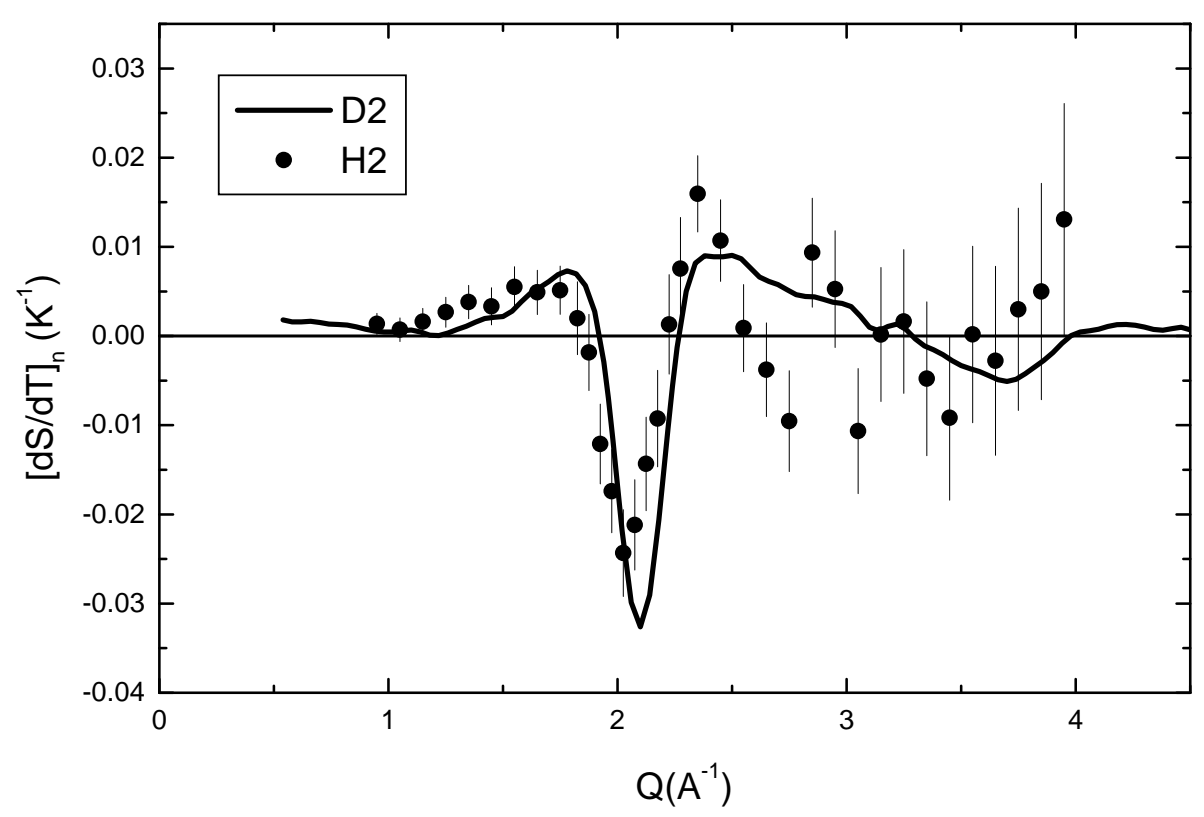

Figure 5. Direct comparison between the temperature derivatives of $S(Q)$ of the hydrogens. The line represents the deuterium behaviour while the hydrogen data are represented by the black dots with the error bars. 
(4 and 5), we can suggest a simple, intuitive, explanation, the difference in the rising edge at small $Q$, that is observed in figure 4 , is totally unexpected. In fact, when the data are plotted in reduced units of $Q$ (i.e. as a function of $Q^{*}=Q \sigma$, where $\sigma$ is the effective molecular diameter), it can be noted that the rising edge and the positive peak of the density derivative, and the whole temperature derivative, become almost coincident provided that the effective diameter of hydrogen is increased by $\sim 2 \%$. As a matter of fact, liquid hydrogen is expected to show larger quantum effects with respect to deuterium. This is consistent with the data reported in table 1 . One aspect of the increased quantum behaviour is an expected increment in the effective molecular size of hydrogen with respect to deuterium. If we reasonably assume that the two isotopes experience the same intermolecular potential, that is determined by the same electronic structure, we note that the observed increase of the effective molecular size is a signature of the expanded single particle wavefunction.

\section{Comparison with the PIMC simulations}

The experimental results have been compared with those of a PIMC simulation carried out in similar thermodynamic conditions. For both hydrogen isotopes, we used the same intermolecular potential, namely the NWB potential [22]. The simulations were carried out using $N=500$ classical particles and Trotter numbers $P$ (= number of bids in the classical isomorphic polymer system) ranging between 1 and 32 [25]. After a suitable number of equilibration moves, starting from a random initial configuration, the various stochastic configurations produced by the PIMC sequence were used to evaluate the thermodynamic averages. Typically, a minimum of $10.000 \times 5 \times N$ moves was used to average the radial distribution function, $g(r)$. The PIMC results were analysed as a function of $P$ and we found that $P=16$ was sufficiently accurate for deuterium, while $P=32$ was sufficiently large to represent the correct quantum limit of hydrogen.

The limited number of particles $(N=500)$ was not so large to produce a simulation $g(r)$ sufficiently extended to obtain a reliable $S(Q)$. In order to extend the cutoff radius from $\approx 14 A$ (i.e. half the size of the simulation box), to a more suitable value of $\approx 30 \mathrm{~A}, N$ should increase by a factor of 10 , and the CPU time would increase, approximately, by two orders of magnitude. Thus, we had to devise a different way of extending the range of $g(r)$, so that we could overcome this limit. To this end, we used an extension procedure, suggested by Verlet [26], which approximates the function $h(r)=g(r)-1$ using a damped oscillating form

$$
h(r)=(A / r) \exp \left(-r / r_{0}\right) \sin \left(r / r_{1}\right) .
$$

The parameters $A, r_{0}$, and $r_{1}$ are obtained by fitting the functional form (5) to the simulation results, starting from the third zero of $h(r)$.

In figures 6 and 7 we report the comparison between the experimental results and the PIMC simulations for the thermodynamic derivatives of the structure factor of the hydrogens. We observe that, for the case of deuterium, there is nice agreement, also quantitative, between the PIMC calculations and the experimental results. For 


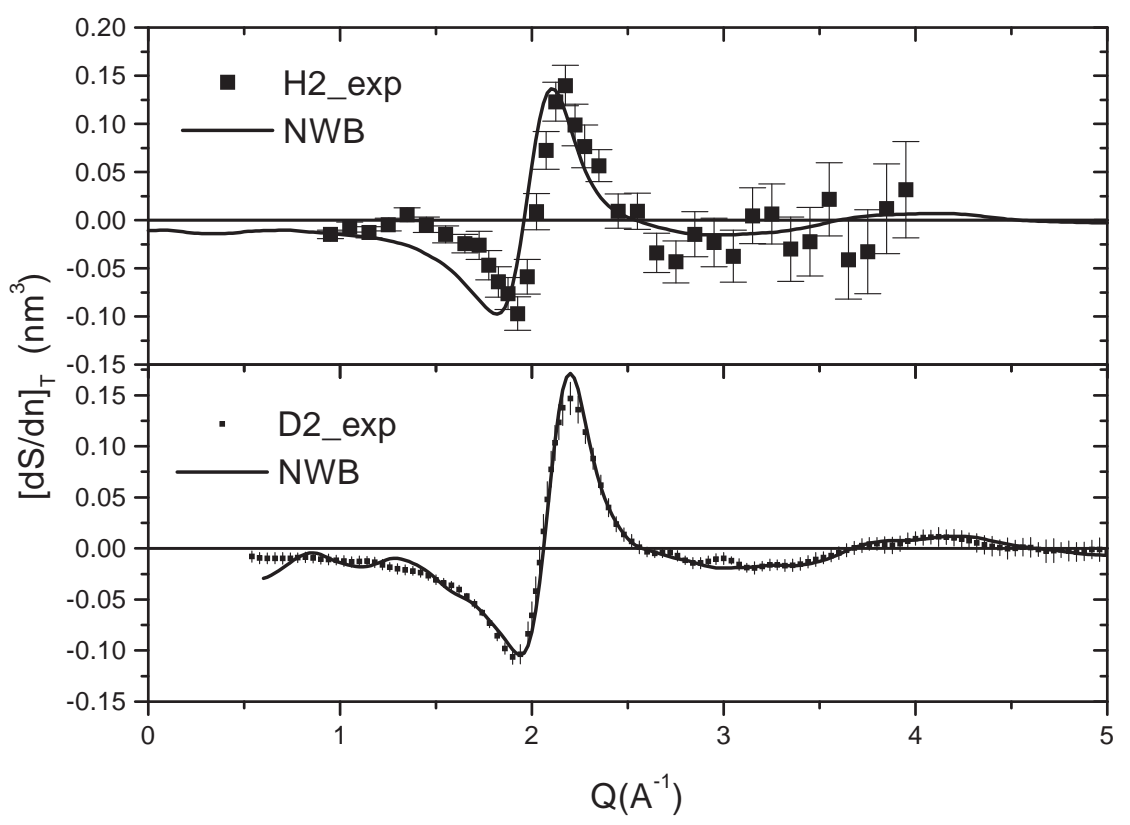

Figure 6. Density derivatives, at constant temperature, of the structure factor of the hydrogens. Comparison between experiment and PIMC simulations using the NWB intermolecular potential. We observe a rather good quantitative agreement for the deuterium data (lower figure) while for hydrogen (upper figure) the agreement cannot be considered more than qualitative.

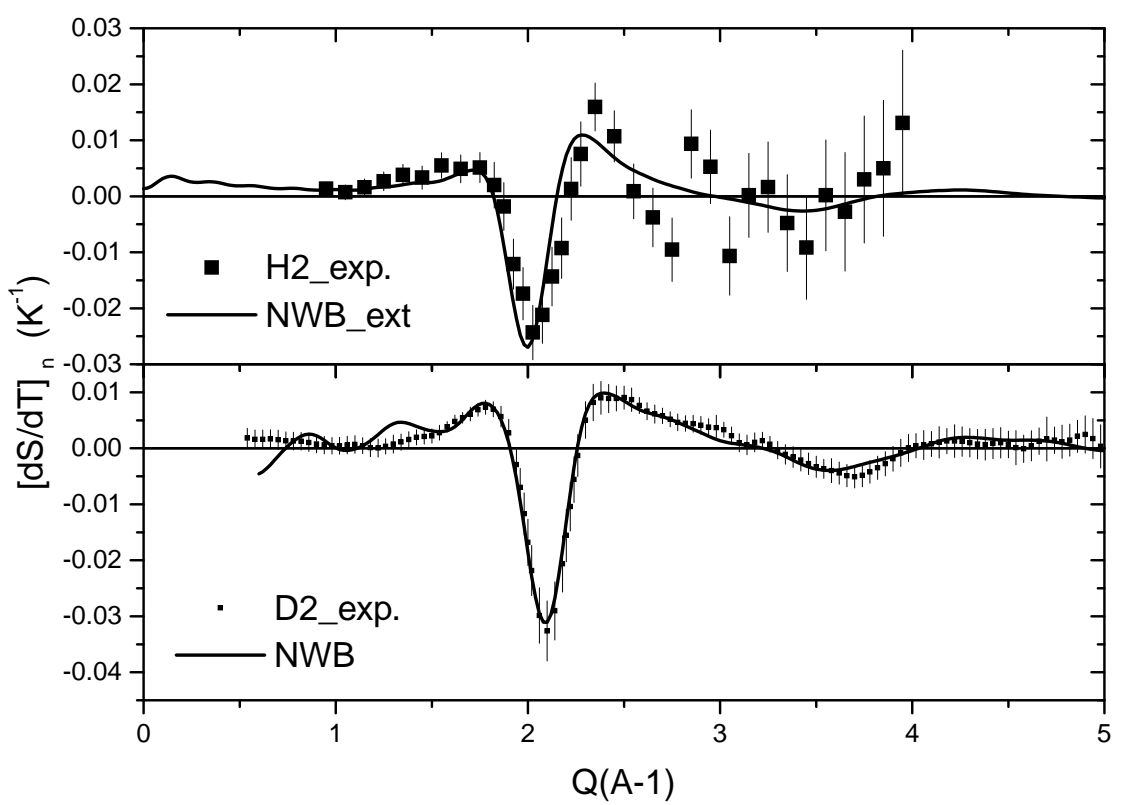

Figure 7. Temperature derivative, at constant density, of the structure factor of the hydrogens. Comparison between experiment and PIMC simulations using the NWB intermolecular potential. We observe a rather good quantitative agreement for the deuterium data (lower figure) while for hydrogen (upper figure) the agreement cannot be considered more than qualitative. 
hydrogen, instead, the agreement is less satisfactory. In the density derivative (figure 6), the data appear a little out-of-phase with respect to the PIMC results. In order to increase the agreement with the simulation, the experimental points should be located to smaller values of $Q$, thus increasing the difference already observed in figure 4 with respect to deuterium. In addition, a further mismatch is observed in the first portion of the experimental function where, a simple shifting of the data is not sufficient to recover the required agreement. A similar qualitative result is observed in figure 7 , but now a simple shift of the simulation results to higher values of $Q$ would appear to be sufficient.

\section{Discussion}

By means of neutron diffraction, we have measured the thermodynamic derivatives of the structure factor of the liquid hydrogens close to the triple point. Due to the relatively small incoherent background we succeeded in obtaining the $S(Q)$ of liquid deuterium. However, no such results could be obtained for hydrogen, due to the combined effect of larger inelasticity effects and an even larger incoherent background. Nevertheless, the thermodynamic derivatives of the microscopic structure factor were obtained in both cases. This experimental information was analysed by itself, by comparing the deuterium and the hydrogen data, and in comparison with the simulation results.

The direct experimental comparison evidenced a different behaviour between light and heavy hydrogen that could be qualitatively attributed to a different size of the hard core diameter of the two molecules. This difference is consistent with a different quantum behaviour related to the mass difference of the two isotopes.

As far as the comparison with the PIMC simulation is concerned, we observe that the simulation results would suggest an even further shift of the hydrogen data to smaller $Q$ values in order to obtain a nice agreement with the experiment. Moreover, a difference in the small- $Q$ interval, already observed in the comparison between the two experimental density derivatives (cf. figure 4) remains unexplained.

The observed differences could be attributed either to residual systematic errors, of unknown origin, that have not been accounted for in the data analysis of the hydrogen experiment, or to the calculated results, especially on going from deuterium to hydrogen. We tend to exclude problems from the simulation technique. In fact, PIMC simulations have been successfully applied to even more quantum systems without problems. However, it may be that the problem stems from the intramolecular model that was used in the data analysis of the experiments. For example, it is possible that the free rotating harmonic oscillator model could be good enough for deuterium but insufficiently accurate for hydrogen.

All we can do here is to further check the experimental data. To this end, we have planned a new experiment, now on a reactor source, to validate the experimental data of liquid hydrogen. In fact, even if this experiment seems to be more difficult, in principle, the expected systematic errors would affect, in this case, a totally different region of $Q$. Therefore, from a critical comparison of the two sets of data, we are 
confident to extract a more precise information.

To sum up, we have shown that the microscopic structure of the hydrogens actually depends, as expected, on the isotopic mass which, in turn, acts on the size of the quantum behaviour of the molecule. Some results are qualitatively consistent with the theoretical predictions, even though a fully satisfactory quantitative agreement hasn't been obtained yet. We hope that further experimental data that will be available in the near future may bring the whole picture to an overall agreement. However, should this not happen, the observed discrepancies would raise a further interesting question concerning the peculiar behaviour of one of the most studied substances on earth and the most abundant, in absolute, in the universe.

\section{References}

1. Balucani U., Zoppi M. Dynamics of the Liquid State. Oxford, Oxford University Press, 1994.

2. Hansen J.P., Mc Donald I. Theory of Simple Liquids. London, Academic Press, 1986.

3. Barocchi F., Neumann M., Zoppi M. // Phys. Rev. A, 1985, vol. 31, p. 4015.

4. Zoppi M., Bafile U., Magli R., Soper A.K. // Phys. Rev. E, 1993, vol. 48, p. 1000.

5. Bermejo F.J., Kinugawa K., Cabrillo C., Bennington S.M., Fak B., FernandezDiaz M.T., Verkerk P., Dawidowski J., Fernandez-Perea R. // Phys. Rev. Lett., 2000, vol. 84, p. 5359.

6. Berne B.J., Thirumalai D. // Ann. Rev. Phys. Chem., 1986, vol. 37, p. 401.

7. Allen M.P., Tildesley D.J. Computer Simulation of Liquids. Oxford, Oxford University Press, 1987.

8. Ceperley D.M. // Rev. Mod. Phys., 1995, vol. 67, p. 279.

9. Van Kranendonk J. Solid Hydrogen. New York, Plenum, 1983.

10. Sears V.F. // Can. J. Phys., 1966, vol. 44, p. 1279.

11. Young J.A., Koppel J.U. // Phys. Rev. A, 1964, vol. 33, p. 603.

12. Zoppi M. // Physica B, 1993, vol. 183, p. 235.

13. Mughabghab S.F., Divadeenam M., Holden N.E. Neutron Cross Sections. New York, Academic Press, 1981.

14. Glyde H.R., Svensson E.C. - In: Methods of Experimental Physics. Edited by Price D.L. and Sköld K. London, Academic, 1987, vol. 23B, Chap. 13 and references therein.

15. de Graaf L.A., Mozer B. // J. Chem. Phys., 1971, vol. 35, p. 4697; BellissentFunel M.C., Buontempo U., Filabozzi A., Petrillo C., Ricci F.P. // Phys. Rev. B, 1992, vol. 45, p. 4605.

16. Andreani C., Dore J., Ricci F.P. // Rep. Prog. Phys., 1991, vol. 54, p. 731.

17. Placzek G. // Phys. Rev., 1952, vol. 86, p. 377.

18. Zoppi M., Magli R., Howells W.S., Soper A.K. // Phys. Rev. A, 1989, vol. 39, p. 4684.

19. Zoppi M., Soper A.K., Magli R., Barocchi F., Bafile U., Ashcroft N.W. // Phys. Rev. E, 1996, vol. 54, p. 2773.

20. Guarini E., Barocchi F., Magli R., Bafile U., Bellissent-Funel M.C. // J. Phys. Condens. Matter, 1995, vol. 7, p. 5777.

21. Zoppi M., Bafile U., Guarini E., Barocchi F., Magli R., Neumann M. // Phys. Rev. Lett., 1995, vol. 75, p. 1779. 
22. Norman M.J., Watts R.O., Buck U. // J. Chem. Phys., 1984, vol. 81, p. 3500.

23. Hirshfelder J.O., Curtiss C.F., Bird R.B. Molecular Theory of Gases and Liquids. New York, Wiley, 1966.

24. Zoppi M., Celli M., Soper A.K. // Phys. Rev. B, 1998, vol. 58, p. 11905.

25. Neumann M., Zoppi M. // Phys. Rev. A, 1991, vol. 44, p. 2474.

26. Verlet L. // Phys. Rev., 1968, vol. 165, p. 201.

\title{
До мікроскопічної структури рідкого водню
}

\author{
М.Зоппі ${ }^{1}$, М.Челлі ${ }^{1}$, У.Бафіле ${ }^{1}$ Е.Гуаріні ${ }^{2}$, М.Нойманн $^{3}$ \\ 1 Інститут квантової електроніки, \\ 1-50127 Флоренція, Італія \\ 2 Національний інститут фізики матерії, \\ Генуя, Італія \\ 3 Інститут експериментальної фізики, \\ А-1090 Відень, Австрія
}

Отримано 23 серпня 2000 р.

Серед квантових рідин водень займає цікаве місце між рідким гелі$€ м$, для якого квантовий обмін приводить до макроскопічного явища надплинності, і неоном, для якого квантові ефекти відносно малі, так що його властивості можуть бути оцінені методами збурень відносно класичної системи. Тим не менше, експериментальний підхід до мікроскопічної структури водню не є легким завданням як через його внутрішню молекулярну структуру, так і через малу молекулярну масу, що співмірна з масою нейтронів. У цій статті ми обговорюємо стан справ і підсумовуємо наявну експериментальну інформацію про мікроскопічну структуру водню. Експериментальні дані для двох систем порівнюються між собою і з результатами розрахунку інтегралів за траєкторіями методом Монте Карло. Знайдено, що подібні величини, виміряні у відповідних термодинамічних точках, є швидше відмінні для двох систем завдяки різним вкладам квантових ефектів. Більше того, порівняння з результатами моделювання показують, що для дейтерію спостерігаються суттєві узгодження як на рівні структурного фактора, так і його термодинамічних похідних. Узгодження $€$ менш задовільним для рідкого водню.

Ключові слова: квантові рідини, рідкий водень, квантові ефекти

PACS: $61.25 . E m, 61.12 . \mathrm{Gz}$ 
